\title{
AFP-producing acinar cell carcinoma treated by pancreaticoduodenectomy in a patient with a previous radical subtotal gastrectomy by gastric cancer
}

\author{
Chang Young Kim${ }^{1}$, Sung Hwan Lee ${ }^{1,3}$, Hyae Min Jeon², Hyun Ki Kim², \\ Chang Moo Kang ${ }^{1,3}$, and Woo Jung Lee ${ }^{1,3}$
}

\begin{abstract}
Departments of ${ }^{1}$ Surgery and ${ }^{2}$ Pathology, Yonsei University College of Medicine, ${ }^{3}$ Pancreaticobiliary Cancer Clinic, Institute of Gastroenterology, Yonsei University Severance Hospital, Seoul, Korea
\end{abstract}

\begin{abstract}
We report a case of alpha-fetoprotein (AFP)-producing acinar cell carcinoma (ACC) of the pancreas. The tumor was diagnosed in a 72 yearold female after radical subtotal gastrectomy (Billroth I) due to early gastric cancer six months before. The initial serum AFP levels were increased to $2,254.1 \mathrm{IU} / \mathrm{ml}$ and preoperative imaging studies showed a mass with approximately $2.5 \mathrm{~cm}$ in diameter near the neck of the pancreas. A pancreaticoduodenectomy was performed. The pathologic examination revealed an ill-defined lobulating tumor confined to the pancreas (T1 stage). Immunohistochemical study showed that the tumor cells expressed AFP. The Adenosine triphosphate-based chemotherapy response assay (ATP-CRA) suggested that cisplatin would be more desirable than gemcitabine in AFP-producing ACC of the pancreas as an adjuvant chemotherapy. However, the adjuvant chemotherapy was not performed due to the early pathological stage. The patient died from carcinomatosis and pneumonia. Even if the tumor was on a relatively early stage, an adjuvant treatment should be considered ACC. (Korean J Hepatobiliary Pancreat Surg 2014;18:33-37)
\end{abstract}

Key Words: Alpha-fetoprotein; Acinar cell carcinoma; Pancreatectomy; Gastric cancer

\section{INTRODUCTION}

The acinar cell carcinoma (ACC) is compared with the ductal cell carcinoma a rare pancreas tumor. ${ }^{1}$ ACCs account for less than $1 \%$ of primary pancreatic neoplasms. ${ }^{2}$ In particular, the pancreatic ACC associated with an elevated serum alpha-fetoprotein (AFP) is extremely rare. ${ }^{3}$ AFP is a glycoprotein with a frequently increase in patients with hepatocellular carcinoma and yolk-sac tumors. ${ }^{4}$ It is not widely known that AFP production can accompany pancreatic ACC. ${ }^{5}$ A standard chemotherapy regimen for AFP-producing ACC has not been established yet. ${ }^{6}$ In this report, a case of resected AFP-producing ACC of the pancreas is presented in a patient who underwent a radical subtotal gastrectomy due to an early gastric cancer. Also it is presented a potential chemotherapeutic regimen based on an in vitro adenosine triphosphate-based chemotherapy response assay (ATP-CRA).

\section{CASE}

A 72-year-old female patient was admitted to our hospital for the evaluation and proper management of a pancreatic mass. Six months ago, she underwent a radical subtotal gastrectomy with gastroduodenostomy (Billroth I) due to early gastric cancer. This pancreatic mass was incidentally identified on a postoperative follow-up abdominal-pelvic computed tomography (CT) scan. Her body weight was $39 \mathrm{~kg}$ and the height was $144 \mathrm{~cm}$ (body mass index, 18.8). A physical examination finding was an upper midline abdominal skin incision in the abdomen, which was soft and flat without palpable mass. All routine blood laboratory tests including amylase and lipase were within normal ranges. Also tumor markers, carcinoembryonic antigen (CEA), carbohydrate antigen 19-9 (CA 19-9) and carbohydrate antigen 125 (CA 125) were within normal limits. In the CT scan, an approximately $2.5 \mathrm{~cm}$-sized

Received: November 15, 2013; Revised: December 10, 2013; Accepted: December 16, 2013

Corresponding author: Chang Moo Kang

Department of Surgery, Yonsei University College of Medicine, Ludlow Faculty Research Building \#204, 50 Yonsei-ro, Seodaemun-gu, Seoul 120-752, Korea

Tel: +82-2-2228-2100, Fax: +82-2-313-8289, E-mail: cmkang@yuhs.ac 
mass near the neck of the pancreas with distal duct dilatation and a parenchyma atrophic change abutting to the portal vein was identified. No lymph node enlargement or distant metastasis was detected (Fig. 1A).The 2-deoxy-2[18F]-fluoro-D-glucose (FDG) positron emission tomography (PET) showed approximately $2.6 \mathrm{~cm}$-sized low-attenuating pancreatic mass in the body portion without significantly increased FDG uptake in any other organs (Fig. 1B). Pancreaticobiliary magnetic resonance imaging with cholangiography showed about $2.5 \mathrm{~cm}$-sized hypervascular, T2 low signal, fat-containing, well-defined mass in the neck area with distal parenchymal atrophy and duct dilatation (Fig. 1C). The characteristics of the tumor seemed to be somewhat different from usual ductal adenocarcinoma of the pancreas, and serum AFP was checked. It was ele- vated to 2,254.1 IU/ml (reference range: 0-7.0 IU/ml).

The patient underwent a pancreaticoduodenectomy (PD). During the laparotomy, malignant ascites, peritoneal seeding or distant metastasis were not found, but severe peritoneal adhesions due to the previous radical subtotal gastrectomy were noted. The anatomic plane was obscured during the PD due to the previous lymph node dissection around the celiac axis, common hepatic artery and the proper hepatic artery. Therefore, a dissection between the neck of the pancreas and superior mesenteric vein-splenic vein-portal vein confluence was needed to identify the anatomic landmark (Fig. 2A). As previous lymph node dissection around major vessels was conducted already, a standard lymph node dissection was performed (Fig. 2B). Due to the previous gastroduodenostomy the reconstruction
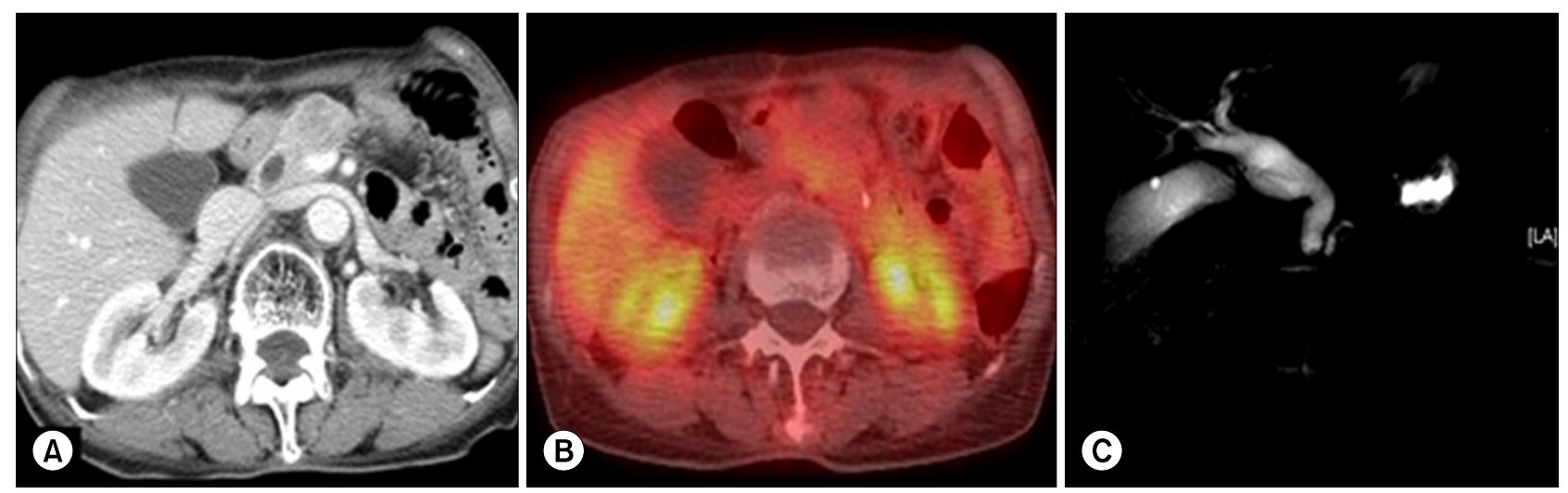

Fig. 1. Preoperative image study findings. CT scan showed about a $2.5 \mathrm{~cm}$-sized mass near the pancreatic neck portion without evidences of distant metastasis (A). No definitive hypermetabolic signal intensity was shown in FDG-PET scan (B). Filling defect and dilatation of the distal pancreatic duct was shown on magnetic resonance cholangiopancreatography (C).
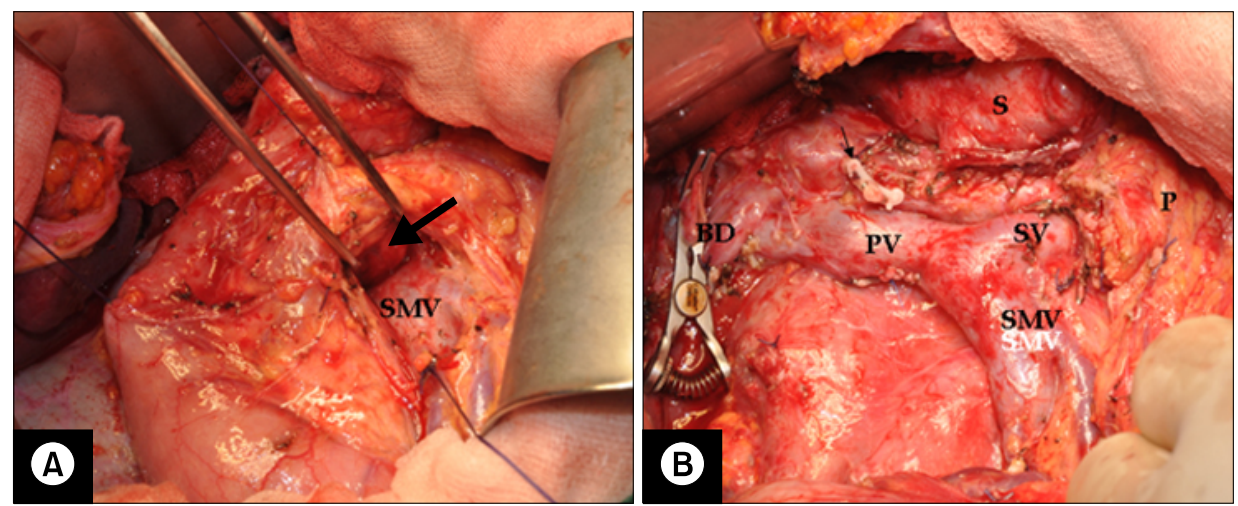

Fig. 2. Intraoperative findings. Severe adhesions were noted, especially around the common hepatic artery and the celiac axis. The pancreatic neck portion needed first to be dissected for the anatomic landmark identification. The round mass at the posterior aspect of the pancreatic neck portion (long, thick arrow) should be noted (A), an operative finding after pancreatoduodenectomy. The stump of the gastroduodenal artery is noted (short, thin arrow) (B). SMV, superior mesenteric vein; SV, splenic vein; PV, portal vein; S, remnant stomach; $\mathrm{P}$, remnant pancreas; $\mathrm{BD}$, common hepatic duct. 
of gastrointestinal continuity was easy following the PD. All procedures, pancreaiticojejunostomy, hepaticojejunostomy and gastrojejunostomy were completed in a usual manner.

An $1.9 \times 1.7 \mathrm{~cm}$-sized ill-defined lobulating tumor confined to the pancreas was revealed in the macroscopic specimen examination (Fig. 3A). An AFP-producing ACC of the pancreas (T1 stage) was classified based on the routine hematoxylin and eosin (H\&E) staining and immunohistochemical staining (Fig. 3B and 3C).

The postoperative recovery course was uneventful. The patient discharged 12 days postoperatively. For the potential possibility of application of chemotherapeutic agents in this rare case of pancreatic cancer, an ATP-CRA chemosensitivity test to anticancer drugs was performed (Table 1). The result showed cisplatin would potentially be more applicable than gemcitabine which is usually used in pancreatic cancer. Although the serum level of AFP had been dropped into normal range immediately after $\mathrm{PD}$, it was rebounded exponentially 17 month postoperative (Fig. 3D). The patient died due to carcinomatosis and pneumonia at 30 months after the surgery.

\section{DISCUSSION}

Benassai et al. $^{7}$ reported that AFP was elevated in only
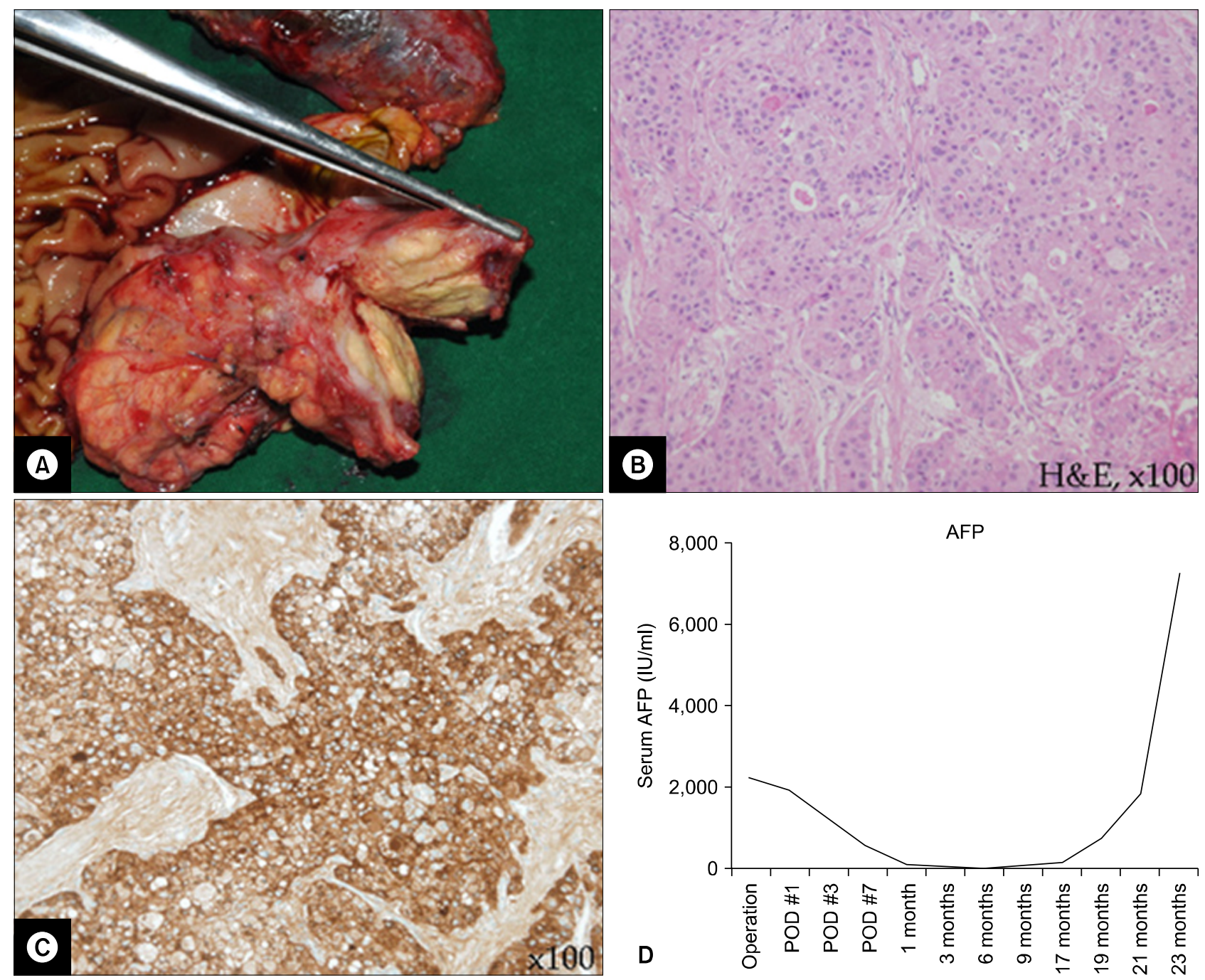

Fig. 3. Surgical pathology and postoperative course. An about $1.9 \mathrm{~cm}$ - sized pancreas-confining lobulated tumor was noted on the neck of the pancreas (A). The tumor cells are arranged in irregular solid sheets and acinar pattern $(\mathrm{B}, \times 100)$, The AFP immunohistochemical staining was diffuse positive in tumor cells $(\mathrm{C}, \times 100)$. The patient got no adjuvant chemotherapy in follow up. The serial follow up of serum AFP showed re-elevation after declining near the normal level following operation (D). H\&E, hematoxylin-eosin. 
Table 1. APT-CRA chemosensitivity report (Tumor inhibition rates at serial concentrations of TDC)

\begin{tabular}{lcccr}
\hline \multirow{2}{*}{\multicolumn{1}{c}{ Drug }} & \multicolumn{3}{c}{ Concentration $(\mathrm{xTDC})$} & \multirow{2}{*}{\begin{tabular}{c} 
Index \\
\cline { 2 - 4 }
\end{tabular}} \\
\cline { 2 - 4 } & $0.2 \mathrm{x}$ & \multicolumn{1}{c}{$1 \mathrm{x}$} & \multicolumn{1}{c}{$5 \mathrm{x}$} & (Rank $\left.^{\dagger}\right)$ \\
\hline Cyclophosphamide & 9.6 & $31.3^{*}$ & $89.1^{*}$ & $170(1)$ \\
Cisplatin & 0 & $44.5^{*}$ & $84.2^{*}$ & $171.3(2)$ \\
Oxaliplatin & $20.7^{*}$ & $32.8^{*}$ & $73.9^{*}$ & $172.6(3)$ \\
5-Fluorouracil & $30.3^{*}$ & $37.8^{*}$ & $58.9^{*}$ & $173(4)$ \\
Doxorubicin & $26^{*}$ & $26.9^{*}$ & $70.8^{*}$ & $176.3(5)$ \\
Gemcitabine & $24.1^{*}$ & $31.2^{*}$ & $50.2^{*}$ & $194.5(6)$ \\
Paclitaxel & 0 & 10.6 & $94.8^{*}$ & $194.6(7)$ \\
Etoposide & 2.9 & $32.3^{*}$ & $69.6^{*}$ & $195.2(8)$ \\
\hline
\end{tabular}

TDC, test drug concentration

${ }^{*} p<0.05$

Index $=300$-sum (of tumor inhibition rate $0.2 \mathrm{x}, 1 \mathrm{x}$ and $5 \mathrm{x}$ )

${ }^{\dagger}$ Rank=more desirable in drug tested

$6 \%$ of ACC patients. Lee et $a{ }^{8}{ }^{8}$ reported that AFP was elevated in only 1 out of 29 Korean ACC patients. Kawamoto et al. ${ }^{9}$ reported that liver metastases were identified in 21 of the 28 patients with AFP-producing pancreatic cancer $(76 \%$ overall). In the most of the reported cases, the AFP-producing ACCs were found to be very aggressive and required systemic chemotherapy. In the reported case, the AFP-producing ACC presented this aggressive nature of malignant characteristics. During the radical subtotal gastrectomy, no pancreatic lesion was found: the tumor needed only 6 month to become a 2.5 cm-sized mass. In this case, resection could be conducted and T1 stage was reported due to the early tumor detection during regular CT scans as follow up after the previous surgery. Therefore an early detection of pancreatic cancers could be suggested due to certain regular screening strategies.

Due to the previous radical subtotal gastrectomy, several issues had to be considered before PD performance in the reported case. First, severe peritoneal adhesions were present, where dexterous skills are needed to expose the retroperitoneal organs adequately. In particular, the previous lymph nodes dissection around the celiac axis, the common hepatic artery and the proper hepatic artery resulted in a dense fibrous adhesion between the pancreas and the subhepatic area. The extensive dissection around these vessels lead to a potential vascular damage related to severe postoperative morbidity because the soft tissues around the major vessels had been removed in previous radical gastrectomy already. Therefore, a standard PD would be an appropriate surgical extent in patients who previously had radical gastrectomy. Last, the gastrointestinal anastomosis after PD is affected by the previous gastrointestinal anastomosis. In the present case, the previous gastroduodenostomy made this reconstruction easy. But in the case of a previous gastrojejunostomy, a different reconstruction strategy needs to be considered.

Gemcitabine is a first-line cancer drug widely used for the chemotherapy in the ductal adenocarcinoma. ${ }^{10}$ However, a chemotherapy regimen for AFP-producing ACC has not been established yet because the number of affected patients is too small. ${ }^{6}$ An in-vitro ATP-CRA (Table 1) was performed by us to evaluate the chemosensitivity of anticancer drugs for the potential application of chemotherapeutic agents in this rare pancreatic cancer case. In recent studies it was found that ATP-CRA results can predict the chemosensitivity in patients with ovarian cancer, gastrointestinal cancer or breast cancer. ${ }^{11-13}$ Our result showed cyclophosphamide is the most desirable drug which was tested for AFP-producing ACC of the pancreas followed by cisplation and gemcitabine is less effective than cyclophosphamide or cisplatin for the AFP-producing ACCs. According to our institutional experience, ${ }^{14}$ ACCs are very rare and show aggressive biologic behaviors such as regional or distant metastasis at the initial time of diagnosis. Currently the best treatment approach is the curative resection according to our institutional experience. ${ }^{14}$ The reported patient received no postoperative adjuvant chemotherapy after the curative pancreatectomy because the tumor was reported as an early pancreatic cancer (T1N0M0, Stage I). However, the patient experienced aggressive carcinomatosis recurrence, cachexia and pulmonary complications, and deteriorated rapidly without application time for adjuvant or palliative chemotherapy. Based on the ATP-CRA, it might have been beneficial to apply adjuvant chemotherapy even if the tumor was on a relative early stage. To determine an effective chemotherapy regimen for AFP-producing ACCs, further experience and studies are necessary.

In summary, the AFP-producing ACC is a very rare malignant pathology in pancreas which requires curative pancreatectomy. Complex difficulties in the PD occurred due to the previous radical subtotal gastrectomy and the surgeon in particular needed to consider the anatomic relationships. Adjuvant postoperative chemotherapy based 
on the results of ATP-CRA test should be considered in this rare and unusual malignancy as well.

\section{REFERENCES}

1. Wisnoski NC, Townsend CM Jr, Nealon WH, et al. 672 patients with acinar cell carcinoma of the pancreas: a population-based comparison to pancreatic adenocarcinoma. Surgery 2008;144: 141-148.

2. Chen J, Baithun SI. Morphological study of 391 cases of exocrine pancreatic tumours with special reference to the classification of exocrine pancreatic carcinoma. J Pathol 1985;146: 17-29.

3. Ishizaki A, Koito K, Namieno T, et al. Acinar cell carcinoma of the pancreas: a rare case of an alpha-fetoprotein-producing cystic tumor. Eur J Radiol 1995;21:58-60.

4. Seregni E, Botti C, Bombardieri E. Biochemical characteristics and clinical applications of alpha-fetoprotein isoforms. Anticancer Res 1995;15:1491-1499.

5. Cingolani N, Shaco-Levy R, Farruggio A, et al. Alpha-fetoprotein production by pancreatic tumors exhibiting acinar cell differentiation: study of five cases, one arising in a mediastinal teratoma. Hum Pathol 2000;31:938-944.

6. Kolb-van Harten P, Rosien U, Klöppel G, et al. Pancreatic acinar cell carcinoma with excessive alpha-fetoprotein expression. Pancreatology 2007;7:370-372.
7. Benassai G, Mastrorilli M, Quarto G, et al. Survival after pancreaticoduodenectomy for ductal adenocarcinoma of the head of the pancreas. Chir Ital 2000;52:263-270.

8. Lee JH, Lee KG, Park HK, et al. Acinar cell carcinoma of the pancreas in Korea--clinicopathologic analysis of 27 patients from korean literature and 2 cases from our hospital--. Korean J Gastroenterol 2010;55:245-251.

9. Kawamoto S, Hiraoka T, Kanemitsu K, et al. Alpha-fetoprotein-producing pancreatic cancer--a case report and review of 28 cases. Hepatogastroenterology 1992;39:282-286.

10. Burris HA 3rd, Moore MJ, Andersen J, et al. Improvements in survival and clinical benefit with gemcitabine as first-line therapy for patients with advanced pancreas cancer: a randomized trial. J Clin Oncol 1997;15:2403-2413.

11. Kawamura H, Ikeda K, Takiyama I, et al. The usefulness of the ATP assay with serum-free culture for chemosensitivity testing of gastrointestinal cancer. Eur J Cancer 1997;33:960-966.

12. Kim HA, Yom CK, Moon BI, et al. The use of an in vitro adenosine triphosphate-based chemotherapy response assay to predict chemotherapeutic response in breast cancer. Breast 2008;17: 19-26.

13. Ng TY, Ngan HY, Cheng DK, et al. Clinical applicability of the ATP cell viability assay as a predictor of chemoresponse in platinum-resistant epithelial ovarian cancer using nonsurgical tumor cell samples. Gynecol Oncol 2000;76:405-408.

14. Kim HU, Ryu JK, Choi SB, et al. The clinical characteristics of acinar cell carcinoma in the Pancreas. Korean J Hepatobiliary Pancreat Surg 2009;13:54-59. 